\title{
Article
}

\section{X-ray Photoemission Spectroscopy Study of Uniaxial Magnetic Anisotropy Induced in a Ni Layer Deposited on a $\mathrm{LiNbO}_{3} \mathrm{Substrate}$}

\author{
Akinobu Yamaguchi ${ }^{1,2, *}$, Takuo Ohkochi ${ }^{2,3}$, Masaki Oura ${ }^{2}{ }^{(0)}$, Keisuke Yamada ${ }^{4}{ }^{\oplus}$, Tsunemasa Saiki $^{5}$, \\ Satoru Suzuki ${ }^{1}$, Yuichi Utsumi ${ }^{1}$ and Aiko Nakao ${ }^{1,6}$ \\ 1 Laboratory of Advanced Science and Technology for Industry, University of Hyogo, 3-1-2 Kouto, Kamigori, \\ Hyogo 678-1205, Japan; ssuzuki@lasti.u-hyogo.ac.jp (S.S.); utsumi@lasti.u-hyogo.ac.jp (Y.U.); \\ a.nakao@aoni.waseda.jp (A.N.) \\ 2 RIKEN SPring-8 Center, 1-1-1 Kouto, Sayo, Hyogo 679-5148, Japan; o-taku@spring8.or.jp (T.O.); \\ oura@spring8.or.jp (M.O.) \\ 3 Japan Synchrotron Radiation Research Institute, 1-1-1 Kouto, Sayo, Hyogo 679-5198, Japan \\ 4 Department of Chemistry and Biomolecular Science, Faculty of Engineering, Gifu University, 1-1 Yanagido, \\ Gifu City, Gifu 501-1193, Japan; yamada_k@gifu-u.ac.jp \\ 5 Manufacturing Technology Department, Hyogo Prefectural Institute of Technology, 3-1-12 Yukihira, Suma, \\ Kobe 654-0037, Japan; saiki@hyogo-kg.jp \\ 6 Research Organization for Nano and Life Innovation, Waseda University, 513 Tsurumaki-cho, Waseda, \\ Shijuku-ku, Tokyo 162-0041, Japan \\ * Correspondence: yamaguti@lasti.u-hyogo.ac.jp
}

check for updates

Citation: Yamaguchi, A.; Ohkochi, T.; Oura, M.; Yamada, K.; Saiki, T.; Suzuki, S.; Utsumi, Y.; Nakao, A. X-ray Photoemission Spectroscopy Study of Uniaxial Magnetic Anisotropy Induced in a Ni Layer Deposited on a $\mathrm{LiNbO}_{3}$ Substrate. Nanomaterials 2021, 11, 1024. https:// doi.org/10.3390/nano11041024

Academic Editor: Jory Yarmoff

Received: 17 March 2021

Accepted: 10 April 2021

Published: 16 April 2021

Publisher's Note: MDPI stays neutral with regard to jurisdictional claims in published maps and institutional affiliations.

\section{Copyright: (c) 2021 by the authors.} Licensee MDPI, Basel, Switzerland. This article is an open access article distributed under the terms and conditions of the Creative Commons Attribution (CC BY) license (https:/ / creativecommons.org/licenses/by/ $4.0 /)$.

\begin{abstract}
The competition between magnetic shape anisotropy and the induced uniaxial magnetic anisotropy in the heterojunction between a ferromagnetic layer and a ferroelectric substrate serves to control magnetic domain structures as well as magnetization reversal characteristics. The uniaxial magnetic anisotropy, originating from the symmetry breaking effect in the heterojunction, plays a significant role in modifying the characteristics of magnetization dynamics. Magnetoelastic phenomena are known to generate uniaxial magnetic anisotropy; however, the interfacial electronic states that may contribute to the uniaxial magnetic anisotropy have not yet been adequately investigated. Here, we report experimental evidence concerning the binding energy change in the ferromagnetic layer/ferroelectric substrate heterojunction using X-ray photoemission spectroscopy. The binding energy shifts, corresponding to the chemical shifts, reveal the binding states near the interface. Our results shed light on the origin of the uniaxial magnetic anisotropy induced from the heterojunction. This knowledge can provide a means for the simultaneous control of magnetism, mechanics, and electronics in a nano/microsystem consisting of ferromagnetic/ferroelectric materials.
\end{abstract}

Keywords: heterojunction; magnetic anisotropy; magnetoelastic effect; $X$-ray photoemission spectroscopy; X-ray magnetic circular dichroism photoemission electron microscopy

\section{Introduction}

The heterojunction, or heterointerface, is of great importance in a wide range of applications, such as thermal-barrier coatings, nanocomposites, electronic and optical devices, giant magnetoresistive and magnetic tunnel junctions, various catalysts, and batteries. In these various applications, for example, in field-effect transistors, solar cells, and light emitting devices, the band alignments of the heterojunction are crucial factors. Novel multiferroic materials [1] might also be created by heterojunctions. The characteristics of a heterojunction vary greatly depending on the surface termination species [2-20]. As pointed out by Stöhr [8], "symmetry breaking and boning at interfaces leads to a variety of anisotropy phenomena in transition metal sandwiches and multilayers". For example, in the $\mathrm{Ni} / \mathrm{Cu}(100), \mathrm{Fe} / \mathrm{Cu}(100)$, and $\mathrm{Fe} / \mathrm{MgO}$ systems, the magnetic anisotropy is induced by the orbital-coupling and spin-flip at the interface $[3,4,17]$. Candidate phenomena that 
are useful for inducing magnetic anisotropy are the Dzyaloshinskii-Moriya interaction (DMI) [21,22], Rashba interaction [23], magnetoelastic effect, and magnetostriction [24,25]. These interactions and effects can also be induced by symmetry breaking and bonding at the interface.

In general, desired magnetic domain structures can be formed by fabricating the films into nano/microstructures, in other words, by properly tailoring the magnetic shape anisotropy [26-30]. In artificial multilayer systems, exchange bias or synthetic antiferromagnetic coupling, which is derived from the quantum interference generated via the heterojunction between ferromagnetic and antiferromagnetic layers, is generally used to control the magnetic properties [7,8,10-18]. As for the uniaxial magnetic anisotropy that arises from the heterojunction, some investigations have been reported in the systems consisting of a thin ferromagnetic layer and a ferroelectric substrate, e.g., lithium niobate $\left(\mathrm{LiNbO}_{3}\right)$ [31-39]. Several of our previous studies have examined unusual magnetic characteristics found in a $30 \mathrm{~nm}$ thick $\mathrm{Ni}$ wire fabricated on a single crystal Y-cut $128^{\circ}$ $\mathrm{LiNbO}_{3}$ substrate [33-36]. Figure 1a shows a photoemission electron microscopy (PEEM) topographical image obtained by an ultraviolet lamp, (Figure 1b,c) X-ray magnetic circular dichroism photoemission electron microscopy (XMCD-PEEM) images at the Ni $L_{3}$-edge, and (Figure 1d) a pair of dichroic X-ray absorption spectra around the Ni $L_{2,3}$-edge of microfabricated $30 \mathrm{~nm}$ thick $\mathrm{Ni}$ patterns on a $\mathrm{LiNbO}_{3}$ substrate, obtained at the soft X-ray beam line BL17SU at SPring-8 [39]. The white and black contrast in Figure 1b,c denote the magnetization aligning parallel and antiparallel to the $X$-ray direction, respectively. We found that uniaxial magnetic anisotropy was induced in the $\mathrm{Ni}$ layer on the $\mathrm{LiNbO}_{3}$ substrate due to the formation of the heterojunction; the uniaxial magnetic anisotropy forced the $\mathrm{Ni}$ magnetizations to stochastically align parallel or antiparallel to the $X$-axis of the $\mathrm{LiNbO}_{3}$ substrate (indicated by an arrow in region (i) of Figure 1b). As previously reported [33-38], a stripe domain structure is naturally formed when the wire is aligned perpendicular to the $\mathrm{X}$-axis of the $\mathrm{LiNbO}_{3}$ substrate, even in the absence of an external magnetic field, due to the competition between magnetic shape anisotropy and uniaxial magnetic anisotropy induced by the heterojunction (region (ii) of Figure 1b) [33-38]. The magnetic domain width is dependent on the width of wire, being almost equal to the width of the wire. The XMCD spectrum of the Ni layer on the $\mathrm{LiNbO}_{3}$ substrate in Figure $1 \mathrm{~d}$ was obtained by taking the difference between the X-ray absorption spectra (XAS) spectra extracted from the white (magnetization parallel to X-ray) and black (antiparallel) regions. These XAS spectra revealed that the Ni layer was not being clearly oxidized. The shape to the XMCD spectrum obtained here was almost the same as that of conventional Ni plain films $[4,9]$. The drastic change in the magnetoresistance effect of the Ni wires elongated perpendicular and parallel to the $X$-axis of the $\mathrm{LiNbO}_{3}$ substrate, as shown in Figure 4 of Ref. [36], reflected the unusual domain structures, as observed in Figure 1.

What induces uniaxial magnetic anisotropy? To investigate the physical origin of uniaxial magnetic anisotropy generated in the $\mathrm{Ni}$ layer fabricated on the $\mathrm{LiNbO}_{3}$ substrate, we performed depth-dependent X-ray photoelectron spectroscopy (XPS). 

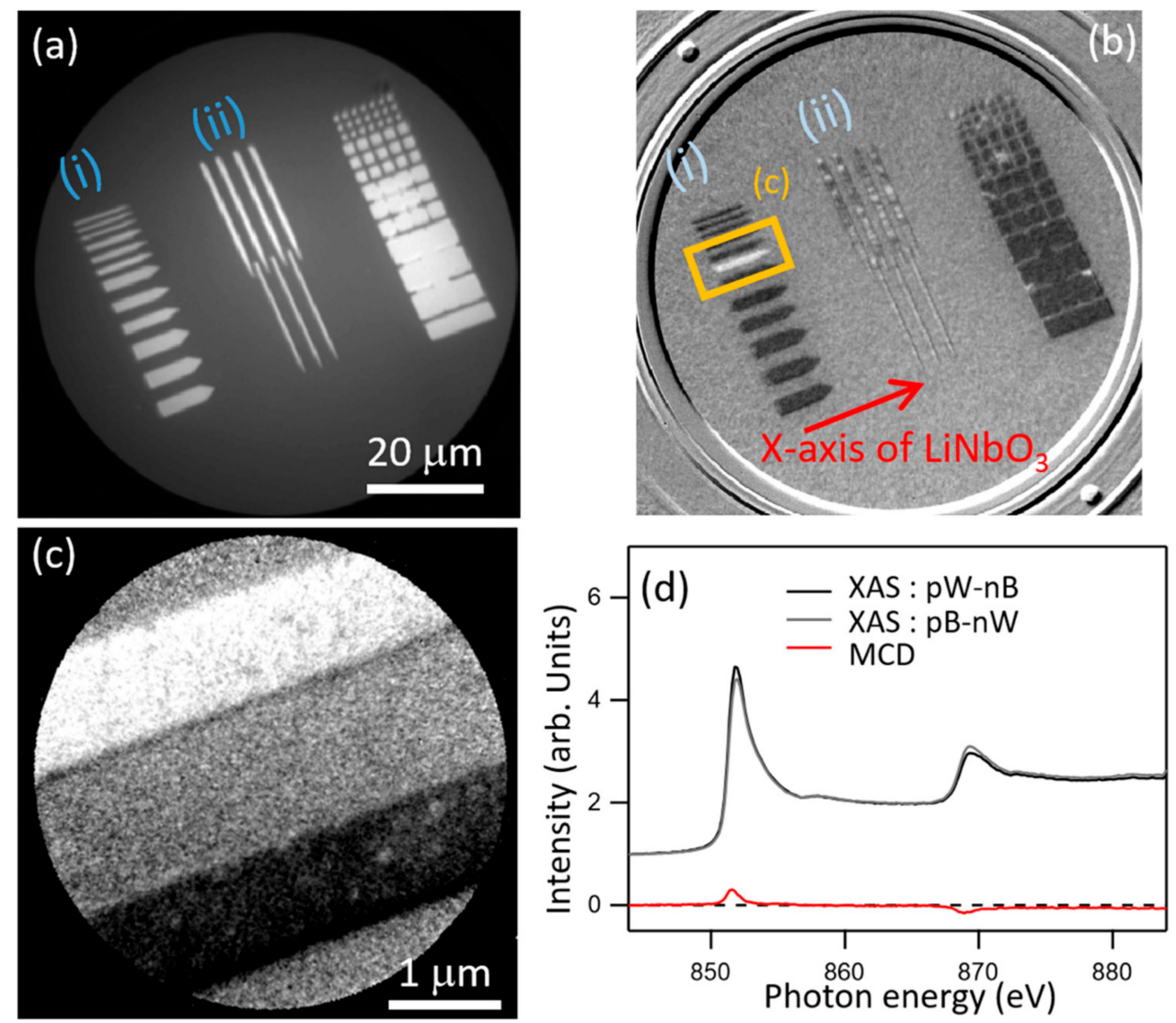

Figure 1. (a) Photoemission electron microscopy (PEEM) topographical image and (b) X-ray magnetic circular dichroism (XMCD)-PEEM image of $30 \mathrm{~nm}$ thick $\mathrm{Ni}$ dots and wires deposited on a Y-cut $128^{\circ} \mathrm{LiNbO}_{3}$ substrate. These dots and wires aligned (i) parallel and (ii) perpendicular to the $X$-axis of the $\mathrm{LiNbO}_{3}$ substrate. (c) Magnified XMCD-PEEM images of Ni wires, whose magnetizations are parallel and antiparallel to the synchrotron radiation light. (d) X-ray absorption spectra (XAS) obtained at the helicity of right $(\mathrm{p})$ and left $(\mathrm{n})$ for the white $(\mathrm{W})$ and black $(\mathrm{B})$ colored contrasts. XAS: $p W-n B$ and XAS: pB-nW are defined as the XAS spectra of intensity $(p W) /(n B)$ and $(p B) /(n W)$, respectively. XMCD spectra of Ni wire calculated from the subtraction of [XAS: $p W-n B]-[X A S$ : pB-nB].

\section{Sample Fabrication and Methods}

Using a magnetron sputtering machine, we deposited several specimens that were composed of a $30 \mathrm{~nm}$ thick Ni layer on a Y-cut $128^{\circ} \mathrm{LiNbO}_{3}$ substrate, or a Si substrate with a naturally oxidized layer formed at the surface, at room temperature $\left(\mathrm{SiO}_{2 \text { (nat) }} / \mathrm{Si}\right)$. The prepared three systems were as follows \#1: Au-cap $(4 \mathrm{~nm}) / \mathrm{Ni}(30 \mathrm{~nm}) / \mathrm{LiNbO}_{3}$, \#2: Au-cap $(4 \mathrm{~nm}) / \mathrm{Ni}(12.5 \mathrm{~nm}) / \mathrm{Au}(12 \mathrm{~nm}) / \mathrm{LiNbO}_{3}$, and \#3: Au-cap $(4 \mathrm{~nm}) / \mathrm{Ni}(12.5 \mathrm{~nm}) / \mathrm{SiO}_{2 \text { (nat) }} / \mathrm{Si}$. In addition, as reference samples, two systems of \#4: $\mathrm{Au}$-cap $(4 \mathrm{~nm}) / \mathrm{Ni}_{81} \mathrm{Fe}_{19}(12.5 \mathrm{~nm}) /$ $\mathrm{LiNbO}_{3}$ and \#5: Au-cap $(4 \mathrm{~nm}) / \mathrm{Ni}_{81} \mathrm{Fe}_{19}(12.5 \mathrm{~nm}) / \mathrm{SiO}_{2 \text { (nat) }} / \mathrm{Si}$ were prepared. The XPS measurements were performed using a VG ESCALAB 250 spectrometer (Thermo Fisher Scientific K.K. Tokyo, Japan), employing monochromatic Al K $\alpha$ X-ray radiation (1486.6 eV) at room temperature. The system was operated at $200 \mathrm{~W}$, and its acceleration voltage was set to be $15 \mathrm{kV}$. The base pressure of the analysis chamber was less than $10^{-8} \mathrm{~Pa}$. A depth profile was measured using an Ar ion sputtering gun with a beam voltage of $3 \mathrm{keV}$. The etching rate was approximately $3 \mathrm{~nm} / \mathrm{min}=0.05 \mathrm{~nm} / \mathrm{s}$ for Si. Even though the etching rate depends on the materials and photoelectron mean free path on the binding energies of the core transitions, we confirmed through a rough estimation that a typical full width of half maximum of a differential of the slope of the XPS intensity $(\sim 40 \mathrm{~s}, \sim 20 \AA)$ was consistent with a convolution of the mean free path $(10 \pm 2-3 \AA$ for the electron kinetic energy of $600-1400 \mathrm{eV}$ ) and the interface roughness of the layered samples (10-20 A) [40]. The XPS 
film thickness dependence (XPS depth profile) was repeatedly measured every time after the Ar sputtering for $\sim 10 \mathrm{~s}$ (the sputtering was paused during the XPS measurements). When the milled surface reached an insulating substrate, a charge balancing treatment was needed. In order to compensate for the surface charge, we used a low-energy electron flood gun. During the course of the electron dozing, a charge neutralizer was referred to in order to over/undercompensate the charge. To further enhance the charge compensation, an earthed conductive grid and a carbon conductive tape were attached around the affected area. In addition, we rechecked the obtained XPS spectra repeatedly to verify reproducibility, including the check of the $\mathrm{Au} 4 f$ and $\mathrm{Ni} 2 p$ peak positions relative to the reference values.

\section{Results and Discussion}

Figure 2a shows the intensity profiles of $\mathrm{Au} 4 f, \mathrm{Ni} 2 p, \mathrm{Nd} 3 d$, and $\mathrm{O} 1 s$ core-level peaks as a function of Ar ion etching time for system \#1. First, the Au $4 f$ peak derived from the Au capping layer appeared for approximately $100 \mathrm{~s}$, which gradually disappeared as the Ni $2 p$ peak increased, that is, as the Ni layer underneath the Au cap was exposed. When approaching the $\mathrm{Ni} / \mathrm{LiNbO}_{3}$ interface (near $1000 \mathrm{~s}$ ), the $\mathrm{O} 1 \mathrm{~s}$ and $\mathrm{Nb} 3 d$ signals appeared while the Ni $2 p$ peak gradually decreased. This etching profile confirmed the correct preparation of the desired system. Next, we focused on the depth dependence of the XPS spectrum shape for each element. Here, let us note that the arrangement of each spectrum was set in order to clarify the temporal transition of the spectral shape. In addition, to track the etching time dependence of the spectrum, some typical etching times are described for the representative spectrum. First, Figure $2 b$ shows the XPS spectra of $\mathrm{Nb} 3 d$ as a function of etching time. According to Refs. [41,42], XPS peaks from $\mathrm{Nb} 3 d$ are decomposed into $3 d 3 / 2$ and $3 d 5 / 2$ contributions: $\mathrm{LiNbO}_{3}(209.41$ and $206.63 \mathrm{eV}), \mathrm{Nb}_{2} \mathrm{O}_{5}$ (209.78 eV, $207.03 \mathrm{eV}), \mathrm{NbO}_{2}(208.23$ and $205.67 \mathrm{eV})$, and $\mathrm{NbO}$ (207.47 and $\left.204.67 \mathrm{eV}\right)$. We found that the subpeak at the binding energy $\left(E_{B}\right)$ of about $204 \mathrm{eV}$ increased with increasing etching time after $1050 \mathrm{~s}$. To understand this behavior and to clarify the mechanism, the fittings were performed in the XPS spectra obtained at $1050 \mathrm{~s}$ and $1300 \mathrm{~s}$, as shown in Figure 2c,d, respectively. As a result, the XPS spectrum at $1050 \mathrm{~s}$ was well-correlated to the fitting calculated by the assumption that these peaks were only the $\mathrm{LiNbO}_{3}$ substrate. The XPS spectrum at $1300 \mathrm{~s}$ was in good agreement with the fitting obtained from the $\mathrm{LiNbO}_{3}, \mathrm{Nb}_{2} \mathrm{O}_{5}$, and $\mathrm{NbO}$. In particular, abrupt increase in the XPS peak at about $204 \mathrm{eV}$ was considered to be derived from $\mathrm{NbO}$. Consequently, the following result was obtained: $\mathrm{NbO}$ was seen just after the pure $\mathrm{Ni}$ layer was removed and the $\mathrm{LiNbO}_{3}$ substrate started to appear ( $\sim 930 \mathrm{~s})$. Then, until the $\mathrm{Ni} / \mathrm{LiNbO}_{3}$ intermixed layer was completely removed ( $1300 \mathrm{~s})$, the XPS intensity of Ni $2 p$ decreased with increasing etching time, as shown in Figure 2e. The $\mathrm{LiNbO}_{3}$ layer partially transformed to $\mathrm{NbO}$ and $\mathrm{Nb}_{2} \mathrm{O}_{5}$-like phases, which were devoid of lithium because the Li was preferentially etched by the Ar+ sputtering [42]. Accompanying the generation of the $\mathrm{NbO}$ and $\mathrm{Nb}_{2} \mathrm{O}_{5}$ XPS peak, the $\mathrm{O} 1 s$ XPS peak also shifted to a lower binding energy, as shown in Figure 2f. Conversely, as shown in Figure 2e, the Ni $2 p$ peak shifted to a higher binding energy as the interface was approached. Note that an XPS peak derived from $\mathrm{NiO}$ was not observed for all depth ranges; we can safely assume that the surface $\mathrm{Ni}$ was perfectly protected against oxidization by the Au cap, and that $\mathrm{Ni}$ hardly reacted with the $\mathrm{LiNbO}_{3}$ substrate. 

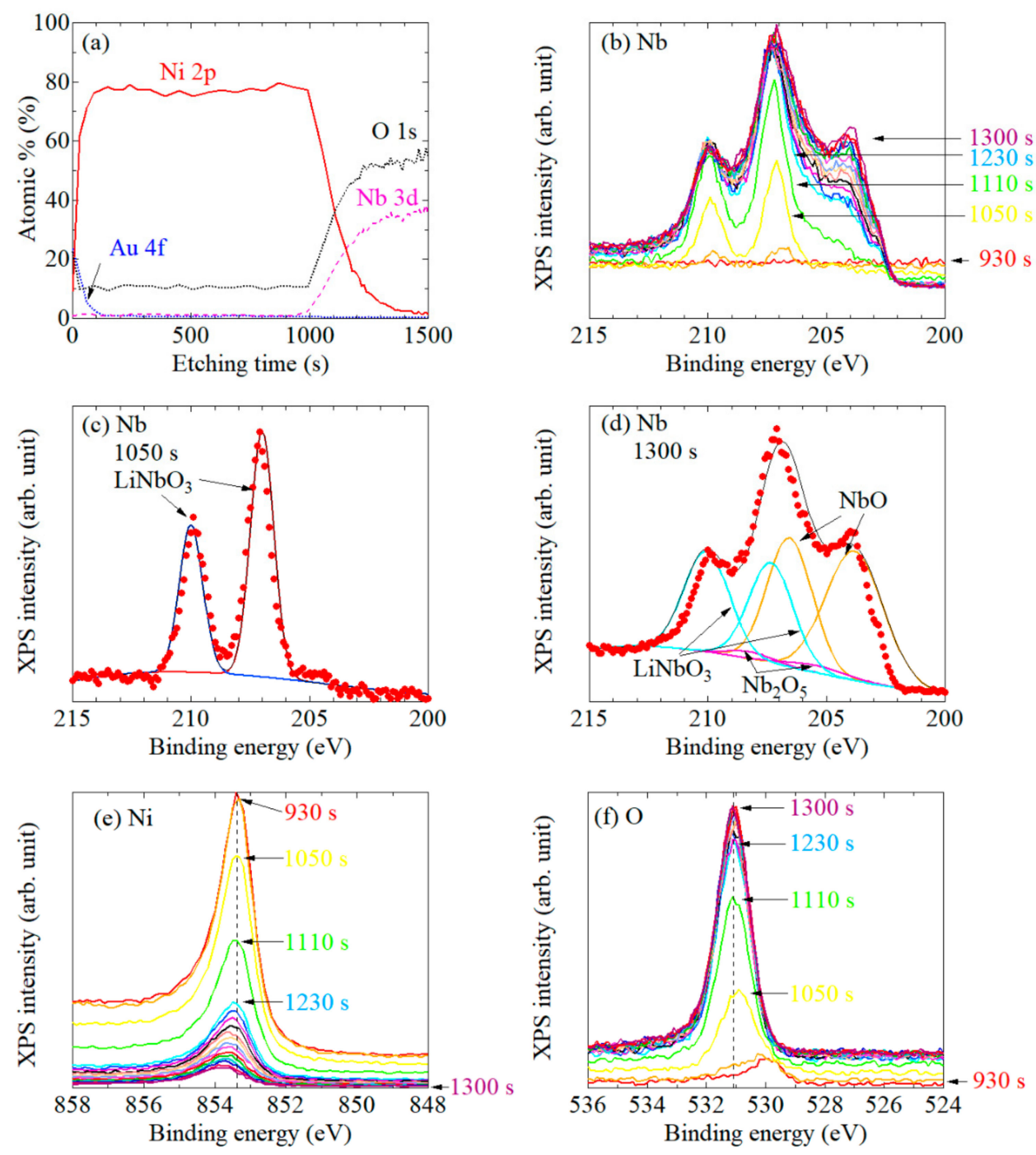

Figure 2. (a) Ar ion etching profiles for the system \#1: the Au-cap/ $\mathrm{Ni} / \mathrm{LiNbO}_{3}$ substrate. XPS spectra of (b-d) $\mathrm{Nb} 3 d$, (e) $\mathrm{Ni} 2 p$, and (f) O 1s. The numbers correspond to the etching times in the etching profile in panel (a). Nd $3 d$ peaks in the XPS spectra at (c) $1050 \mathrm{~s}$ and (d) $1300 \mathrm{~s}$ are fitted. For clarity, the spectra are vertically shifted. The arrangement of each spectrum is set to the forward direction or the reverse direction for each etching time. Hereinafter, in all the figures, a similar drawing is used.

To confirm the Ni film thickness dependence of the XPS spectra, the same measurement was performed for a sample with the same multilayer configuration as \#1, but the $\mathrm{Ni}$ thickness was changed to $12.5 \mathrm{~nm}$. The overall trend in the XPS spectra was practically the same as that shown in Figure 2a-f (not shown here), in that the XPS peak of Ni $2 p$ always shifted to a higher binding energy near the interface with the $\mathrm{LiNbO}_{3}$ substrate. This result suggests that the electron state can only be modulated around the interface, as long as the $\mathrm{Ni}$ thickness is at least thicker than $12.5 \mathrm{~nm}$.

Next, in a similar procedure, we measured the XPS spectra of system \#2: the Aucap $/ \mathrm{Ni} / \mathrm{Au} / \mathrm{LiNbO}_{3}$ substrate, to investigate the interface effect. The intensity profiles of the XPS peaks, together with the variation in the shapes of the Au $4 f, \mathrm{Ni} 2 p$, and O $1 s$ spectra with respect to the etching time, are shown in Figure 3a-d. No shift in the Au $4 f$ XPS spectra was observed for both sides of the interlayer with the Ni layer and the $\mathrm{LiNbO}_{3}$ substrate (Figure $3 b$ ), while the Ni $2 p$ XPS peak shifted to a lower binding energy as the Ni approached the Au layer (Figure 3c). The trend in the Ni $2 p$ peak shift was opposite to the case of the system consisting of the Au-cap/Ni/LiNbO 3 substrate, as shown in Figure 2e. 
This result indicates that the combination of materials at the interface plays a significant role in the modulation of the bonding state of the interfacial $\mathrm{Ni}$.
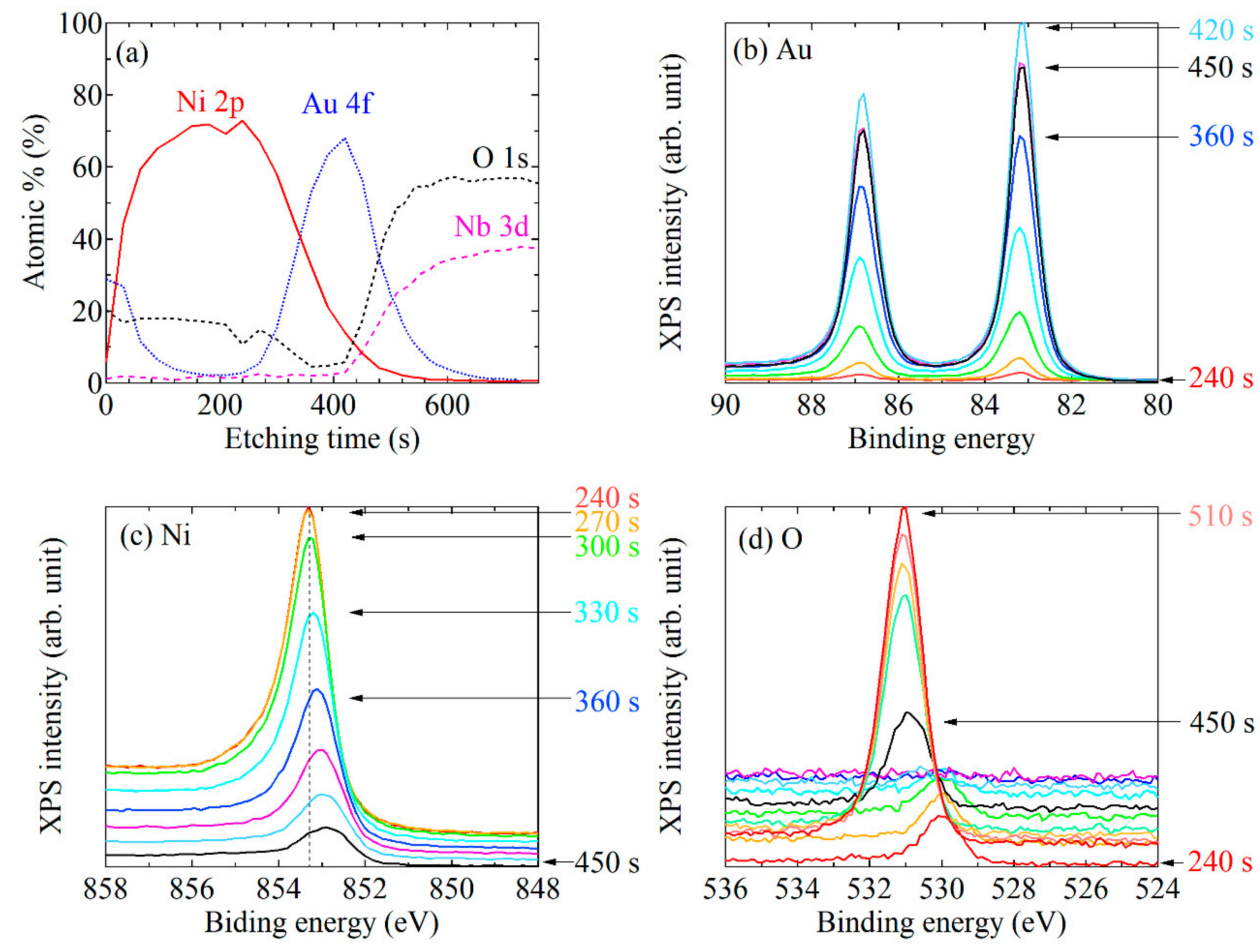

Figure 3. (a) Ar ion etching profiles for the system \#2: the Au-cap/Ni/Au/ $\mathrm{LiNbO}_{3}$ substrate. XPS spectra of (b) Au 4f, (c) Ni 2p, and (d) O 1 s.

To investigate the substrate dependence of the XPS spectra, we measured the XPS of a system consisting of an Au capping layer and a Ni layer deposited on a slightly oxidized Si substrate (\#3). The etching profile is shown in Figure 4a. The XPS spectra of Ni $2 p$ are displayed in Figure $4 \mathrm{~b}$. As shown in Figure $4 \mathrm{~b}$, we found that the Ni $2 p$ XPS spectra abruptly changed between $340 \mathrm{~s}$ and $380 \mathrm{~s}$, near the interface. The newly appeared peak at approximately $855 \mathrm{eV}$, being a higher binding energy than the Ni $2 p$ fundamental peak position, corresponded to the peak derived from $\mathrm{Ni}$ oxide $(\mathrm{NiO})$. When approaching the interface and surface of Si substrate, $\mathrm{Ni} 2 p$ metallic peak almost disappeared after $420 \mathrm{~s}$. Next, we checked the XPS spectra of O 1s and Si 1s. Figure 4c,d indicates that the XPS peak positions of $\mathrm{O} 1 \mathrm{~s}$ and $\mathrm{Si} 1 \mathrm{~s}$ also shifted, synchronizing with the $\mathrm{NiO}$ forming process. This result indicates that the oxygen at the $\mathrm{Ni} / \mathrm{SiO}$ interface preferentially bonds with nickel, which results in the formation of $\mathrm{NiO}$, even if the oxidized $\mathrm{Si}$ layer is vanishingly thin (unlike the case of thermally oxidized thick $\mathrm{SiO}_{2}$ ). $\mathrm{NiO}$ is a well-known antiferromagnetic material. These results indicate that the $\mathrm{NiO}$ formed at the interface might act as a pinning layer against the magnetic domain wall displacement, or the magnetization reversal. 

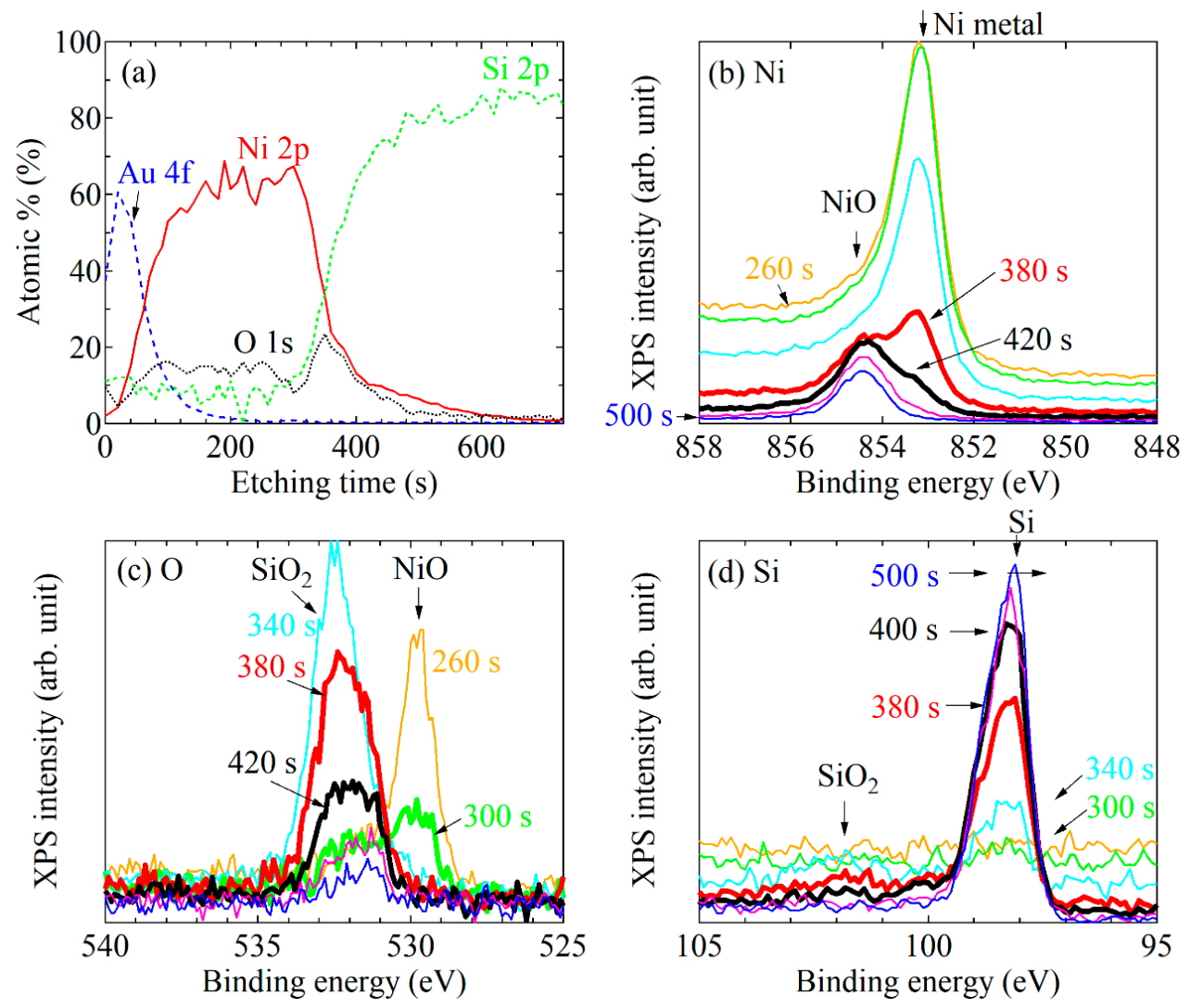

Figure 4. (a) Ar ion etching profiles of the system \#3: the Au-cap/Ni/naturally oxidized $\mathrm{SiO}_{2} / \mathrm{Si}$ substrate. XPS spectra of (b) Ni $2 p$, (c) O 1s, and (d) Si $2 p$.

Next, to compare the XPS spectra of $\mathrm{Ni}_{81} \mathrm{Fe}_{19}$ layers on $\mathrm{LiNbO}_{3}$ and $\mathrm{Si}$ substrates with that of Ni layers on these substrates, we measured the XPS spectra of systems \#4 and \#5 in a similar way. As seen from the intensity profiles in Figure 5a, the spectrum of each element around $400 \mathrm{~s}$ would be useful to closely investigate the $\mathrm{Ni}_{81} \mathrm{Fe}_{19} / \mathrm{LiNbO}_{3}$ interface states. The shape of the Fe $2 p$ spectra shown in Figure $5 \mathrm{~b}$ remained unchanged through all the depth range, although the overall peak intensity decreased as the $\mathrm{Ni}_{81} \mathrm{Fe}_{19}$ layer was etched out. The XPS peak positions of Ni $2 p$ (Figure $5 \mathrm{c}$ ) and O 1 (Figure $5 \mathrm{~d}$ ) slightly depended on the etching time. In the $\mathrm{O} 1 s$, the peak position slightly shifted toward binding energy during 330-420 s, and then settled to $E_{B} \sim 531 \mathrm{eV}$. The Ni $2 p$ peak appeared to shift to the higher binding energy when approaching the substrate; however, it was subtler than the case of $\mathrm{Ni} / \mathrm{LiNbO}_{3}$ (Figure 2c) and Ni/Au interfaces (Figure 3c). These results indicate that the bonding state of $\mathrm{Ni}_{81} \mathrm{Fe}_{19}$ layer is not strongly influenced by the $\mathrm{LiNbO}_{3}$ substrate.

Figure 6 shows similar plots to Figures $2-5$ for the systems \#5: $\mathrm{Ni}_{81} \mathrm{Fe}_{19}$ deposited on a naturally oxidized $\mathrm{Si}$ substrate. Ni $2 p$ spectrum (Figure $6 \mathrm{~b}$ ) shows a single peak indicative of metallic $\mathrm{Ni}\left(E_{\mathrm{B}}=853.5 \mathrm{eV}\right)$ until the surface reached $\mathrm{Ni} / \mathrm{SiO}_{x}$ interface $(<350 \mathrm{~s})$ but the subpeak that derived from $\mathrm{NiO}\left(E_{\mathrm{B}}=854.7 \mathrm{eV}\right)$ became dominant in the middle of the interface ( $>420 \mathrm{~s}$ ). The XPS spectra of Si tended to drastically change during $330-390 \mathrm{~s}$, as shown in Figure 6d. The $\mathrm{O} 1 \mathrm{~s}$ spectrum (Figure 6c) showed a tiny peak at $\sim 530 \mathrm{eV}$ before reaching the interface $(330 \mathrm{~s})$, then it grew and settled to the fixed position of $532.5 \mathrm{eV}$, where we could not distinguish the attribution $\left(\mathrm{NiO}\right.$ or $\left.\mathrm{SiO}_{2}\right)$, but presumably the oxygen was almost fully transferred to the $\mathrm{Ni}$ (or $\mathrm{Fe}$ ) site, considering that the peak attributable to $\mathrm{SiO}_{2}\left(E_{\mathrm{B}}=103-104 \mathrm{eV}\right)$ was not existent through all the temporal range in the $\mathrm{Si} 1 \mathrm{~s}$ spectrum (Figure 6d). 

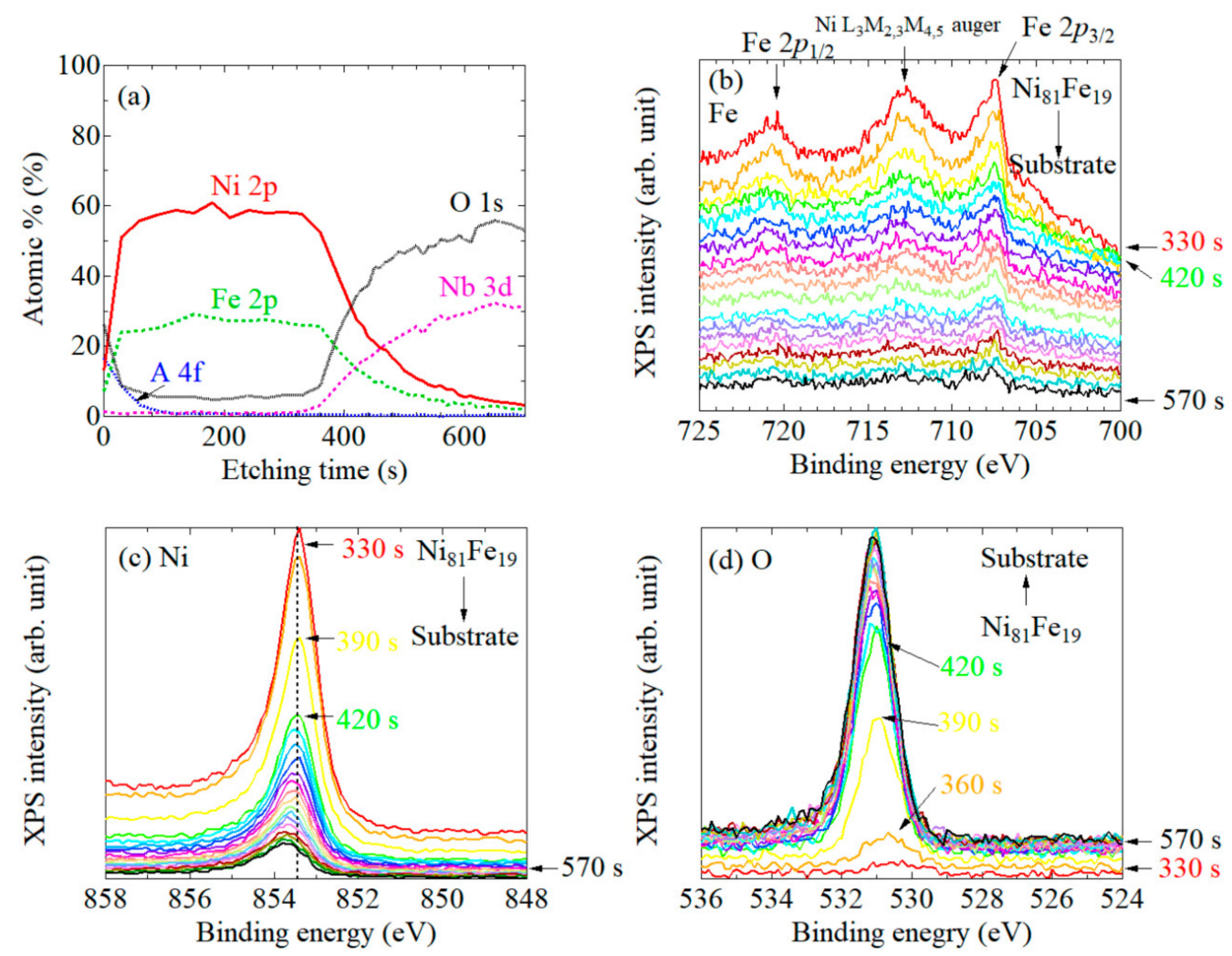

Figure 5. (a) Ar ion etching profiles of the system \#4: the Au-cap $/ \mathrm{Ni}_{81} \mathrm{Fe}_{19} / \mathrm{LiNbO}_{3}$ substrate. XPS spectra of (b) Fe $2 p$, (c) Ni $2 p$, and (d) O 1 s.

Therefore, it appears that the binding strength of $\mathrm{Ni}$ with respect to $\mathrm{O}$ is large and hence the formation of a $\mathrm{NiO}$ layer is inevitable at the interface between the $\mathrm{Ni}$ layer and the (naturally) oxidized Si substrate. This is important when investigating the pinning site against the domain wall displacement in a system consisting of a $\mathrm{Ni}$ film and a $\mathrm{SiO}_{2}$ (or a $\mathrm{Si}$ ) substrate. In contrast, as is obvious from the cases of the systems \#1 and \#3, the Ni layer is not oxidized at the interface between $\mathrm{Ni}$ and the $\mathrm{LiNbO}_{3}$ substrate. In other words, the oxygen in the $\mathrm{LiNbO}_{3}$ is very stable and rarely causes chemical reaction with contacting layers such as $\mathrm{Ni}$. Therefore, $\mathrm{LiNbO}_{3}$ does not lose its piezoelectric feature, which in turn conveys strong uniaxial magnetic anisotropy to the adjacent magnetic layer.

Here, we discuss the systematic peak shifts in the Ni $2 p$ core photoemission that occurred in samples \#1 and \#2, that is, the Ni $2 p$ peak shifted to a lower binding energy when approaching the interface of $\mathrm{Ni} / \mathrm{Au}$ interface, whereas it went to a higher binding energy in the case of $\mathrm{Ni} / \mathrm{LiNbO}_{3}$ interface. Because the $\mathrm{Ni}$, which is in contact with an oxide substrate, tends to firmly attach with each other by a Coulomb attractive force if not chemically reacted to the point of forming $\mathrm{NiO}$, the outer shell electrons of the $\mathrm{Ni}$ are slightly attracted to the oxygen site, which leads to the increase in the binding energy of the core electrons. In the case of the $\mathrm{Ni}$ in the vicinity of a noble metal like $\mathrm{Au}$, by contrast, the $\mathrm{Ni}$ on the Au side form a dangling-bond and hence the binding energy of the $2 p$ core tends to be weakened. Summarizing the above speculations, the $\mathrm{Ni}$ and the $\mathrm{LiNbO}_{3}$ layers are adhesive to each other by indirect $\mathrm{Ni}-\mathrm{O}$ Coulomb interaction, while they do not chemically react with each other and the $\mathrm{LiNbO}_{3}$ maintains the bulk property (piezoelectric material) even at the surface/interface. Therefore, the strong uniaxial anisotropy at the $\mathrm{Ni} / \mathrm{LiNbO}_{3}$ interface may be induced because the magnetoelastic effect due to the crystallographic striction of the ferroelectric $\mathrm{LiNbO}_{3}$ is conveyed to the Ni layer quite effectively. 

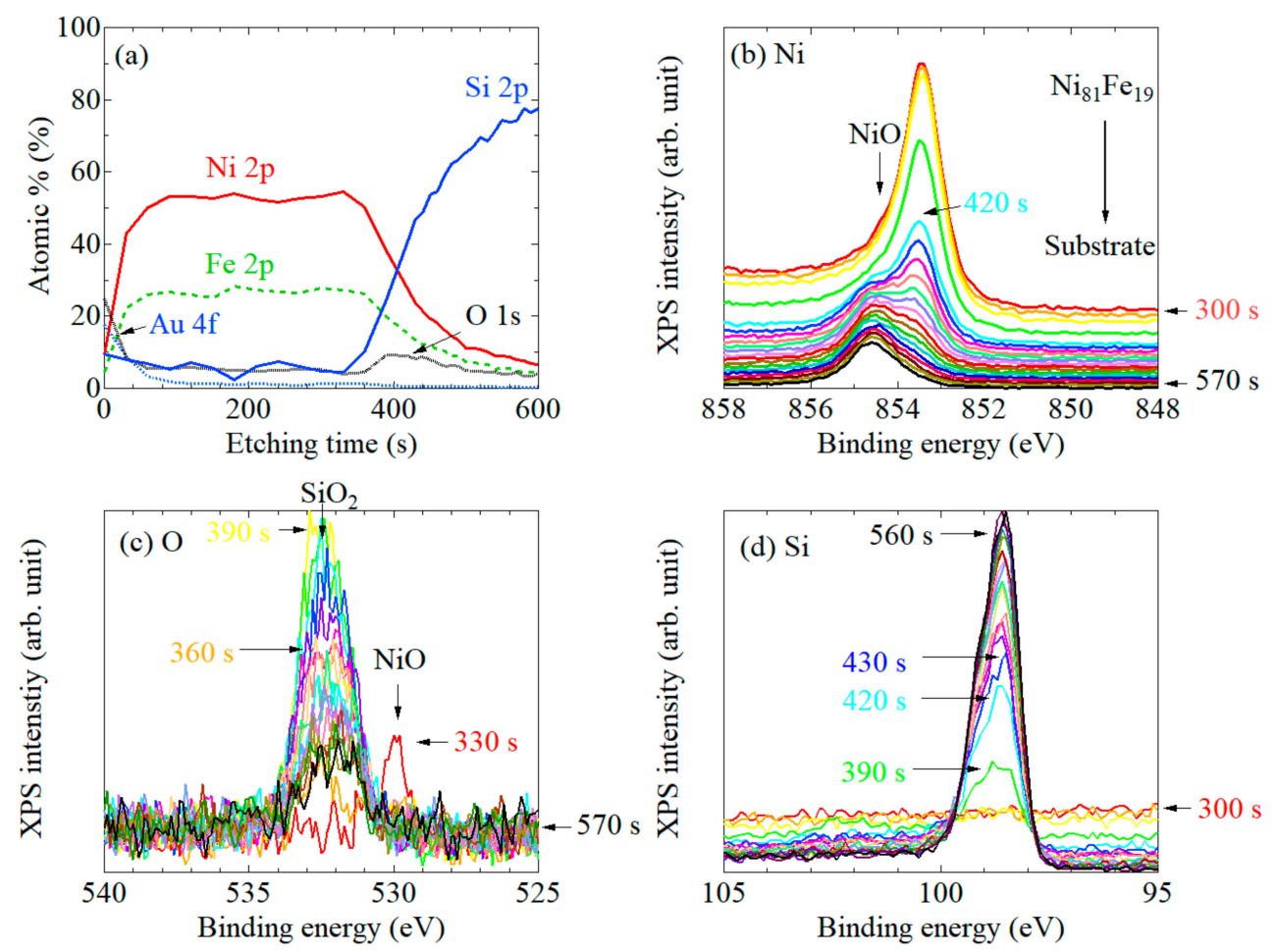

Figure 6. (a) Ar ion etching profiles of the system \#5: the Au-cap/ $\mathrm{Ni}_{81} \mathrm{Fe}_{19} /$ naturally oxidized $\mathrm{SiO}_{2} / \mathrm{Si}$ substrate. XPS spectra of (b) Ni $2 p$, (c) O 1s, and (d) $\mathrm{Si} 2 p$.

Therefore, the heterojunction between the magnetic metallic film and the ferroelectric substrate induces the uniaxial magnetic anisotropy via the interface effect including the magnetoelastic effect, magnetostriction [5,24,25], DMI [21,22], and electron wave function interference at the interface through the Rashba effect [23]. This investigation revealed an electronic state modulated by the heterojunction. This modulation of the electronic state via the heterojunction enables the generation of magnetic anisotropy. As a result, the combination of the shape magnetic anisotropy and uniaxial anisotropy induced by the heterojunction enables the magnetic domain structure formation in microscale $\mathrm{Ni}$ wires fabricated on a $\mathrm{LiNbO}_{3}$ substrate to be controlled. The voltage application of the system consisting of ferroelectric and ferromagnetic materials might enable us to control the magnetization reversal and magnetic anisotropy $[31,32,37,38]$.

\section{Conclusions}

We conducted XPS analyses to unveil the mechanism of the uniaxial magnetic anisotropy generation in $\mathrm{Ni}$ deposited on $\mathrm{LiNbO}_{3}$ substrates. Fine structures of the XPS spectra vary depending on the combination of the interface materials. The XPS results revealed the biding energy modulation of $\mathrm{Ni}$ layer on $\mathrm{LiNbO}_{3}$, and the formation of $\mathrm{NiO}$ layer on naturally oxidized $\mathrm{Si}$ and $\mathrm{SiO}_{2} / \mathrm{Si}$ substrates. These changes in the XPS spectra indicated that the unusual uniaxial magnetic anisotropy generated in the $\mathrm{Ni} / \mathrm{LiNbO}_{3}$ system effectively induced by the internal stress and band modulation associated with the heterojunction formation. We found that the $\mathrm{Ni} / \mathrm{LiNbO}_{3}$ heterojunction was fairly firm but never formed any weird interface materials by chemical reactions without annealing. In addition, a $\mathrm{NiO}$ layer always formed at the interface between $\mathrm{Ni}$ and $\mathrm{SiO}_{2}$. These results indicate that the symmetry of the layer structure and, in turn, the magnetic anisotropy of the film are strongly linked to the ferroelectric substrate, and chemical reactions are unavoidable near the interface depending on the composition of the films. These chemical couplings near the interface between ferromagnetic and ferroelectric layers are critically important to understanding the physical and chemical mechanisms causing functional properties. From the viewpoint of the material design, our study provides valuable information. The 
formation of a heterojunction may be a promising technique to develop novel functional materials.

Author Contributions: A.Y. considered this study and prepared the sample for the XMCD-PEEM and XPS measurements with T.S., T.O., K.Y. and M.O. performed the XMCD-PEEM experiments and analyzed the data. A.N. performed the XPS measurements. A.Y. analyzed the XPS data with K.Y., S.S., Y.U. and A.N., A.Y. wrote the manuscript under discussion with T.O., K.Y., M.O., A.N., and all the authors discussed the results and commented on the manuscript. All authors have read and agreed to the published version of the manuscript.

Funding: This work was supported in part by JSPS Grants-in-Aid for Scientific Research B (No. 17H02755) and the Shimadzu science and technology foundation. The experiments at the BL17SU beamline of SPring- 8 were performed with the approval and support of the Japan Synchrotron Radiation Research Institute (JASRI, Proposal Nos. 2018A1317, 2019A1404, and 2019B1345).

Acknowledgments: We thank Yukako Takizawa of Hyogo Prefectural Institute of Technology for the experimental support.

Conflicts of Interest: The authors declare no conflict of interest.

\section{References}

1. Fiebig, M.; Lottermoser, T.; Meier, D.; Trassin, M. The evolution of multiferroics. Nat. Rev. Mater. 2016, 1, 16046. [CrossRef]

2. Moormann, H.; Kohl, D.; Heiland, G. Work function and band bending on clean cleaved zinc oxide surfaces. Surf. Sci. 1979, 80, 261. [CrossRef]

3. Amemiya, K.; Kitagawa, S.; Matsumura, D.; Abe, H.; Ohta, T.; Yokoyama, T. Direct observation of magnetic depth profiles of thin Fe films on $\mathrm{Cu}(100)$ and $\mathrm{Ni} / \mathrm{Cu}(100)$ with the depth-resolved x-ray magnetic circular dichroism. Appl. Phys. Lett. 2004, 84, 936. [CrossRef]

4. Amemiya, K.; Sakai, E.; Matsumura, D.; Abe, H.; Ohta, T.; Yokoyama, T. Spin-reorientation transition of Ni/Cu(100) and $\mathrm{CO} / \mathrm{Ni} / \mathrm{Cu}(100)$ : Separation of the surface and bulk components of the $\mathrm{x}$-ray magnetic circular dichroism spectrum. Phys. Rev. B 2005, 71, 214420. [CrossRef]

5. Okabayashi, J.; Miura, Y.; Taniyama, T. Strain-induced reversible manipulation of orbital magnetic moments in Ni/Cu multilayers on ferroelectric $\mathrm{BaTiO}_{3}$. NPJ Quantum Mater. 2019, 4, 21. [CrossRef]

6. Okabayashi, J.; Koo, J.W.; Sukegawa, H.; Mitani, S.; Takagi, Y.; Yokoyama, T. Perpendicular magnetic anisotropy at the interface between ultrathin Fe film and $\mathrm{MgO}$ studied by angular-dependent x-ray magnetic circular dichroism. Appl. Phys. Lett. 2014, 105, 122408. [CrossRef]

7. Nogués, J.; Schuller, I.K. Exchange bias. J. Magn. Magn. Mater. 1999, 192, 203. [CrossRef]

8. Stöhr, J.; Anders, S. X-ray spectro-microscopy of complex materials and surfaces. IBM J. Res. Develop. 2000, 44, 536. [CrossRef]

9. Duba, L.; Stöhr, J.; Mancini, D.C.; Nilsson, A.; Wassdahl, N.; Nordgren, J.; Samant, M.G. Magnetic dichroism in $L_{2,3}$ emission of $\mathrm{Fe}, \mathrm{Co}$, and Ni following energy-dependent excitation with circularly polarized x-rays. Phys. Rev. B 1994, $50,16758$.

10. Kim, K.; Lee, K.-J.; Lee, H.; Stiles, M.D. Perpendicular magnetic anisotropy of two-dimensional Rashba ferromagnet. Phys. Rev. B 2016, 94, 184402. [CrossRef]

11. Pradipto, A.M.; Yakushuji, K.; Ham, W.S.; Kim, S.; Shiota, Y.; Moriyama, T.; Kim, K.; Lee, H.; Nakamura, K.; Lee, K.; et al. Enhanced perpendicular magnetocrystalline anisotropy energy in an artificial magnetic material with bulk spin-momentum coupling. Phys. Rev. B 2019, 99, 108410(R). [CrossRef]

12. Yin, Y.; Han, D.; Kim, J.; Lavrijsen, R.; Lee, K.; Lee, S.; Kim, K.; Lee, H.; Swagten, H.J.M.; Koopmans, B. Chiral magnetoresistance in Pt/Co/Pt zigzag wires. Appl. Phys. Lett. 2017, 110, 122401. [CrossRef]

13. Grünberg, P.; Shreiber, R.; Pang, Y.; Brodsky, M.B.; Sowers, H. Layered Magnetic Structures: Evidence for Antiferromagnetic Coupling of Fe Layers across Cr Interlayers. Phys. Rev. Lett. 1986, 57, 2442. [CrossRef]

14. Baibich, M.N.; Broto, J.M.; Fert, A.; van Dau, F.N.; Petroff, F.; Etienne, P.; Creuzet, G.; Friederich, A.; Chazelas, J. Giant Magnetoresistance of (001)Fe/(001)Cr Magnetic Superlattices. Phys. Rev. Lett. 1988, 61, 2472. [CrossRef]

15. Parkin, S.S.; Bhadra, R.; Roche, K.P. Oscillatory magnetic exchange coupling through thin copper layers. Phys. Rev. Lett. 1991, 66, 2152. [CrossRef]

16. Miyazaki, T.; Tezuka, N. Giant magnetic tunneling effect in $\mathrm{Fe} / \mathrm{Al}_{2} \mathrm{O}_{3} / \mathrm{Fe}$ junction. J. Magn. Magn. Mater. 1994, 139 , L231. [CrossRef]

17. Yuasa, S.; Fukushima, A.; Nagahama, T.; Ando, K.; Suzuki, Y. High Tunnel Magnetoresistance at Room Temperature in Fully Epitaxial Fe/MgO/Fe Tunnel Junctions due to Coherent Spin-Polarized Tunneling. Jpn. J. Appl. Phys. 2004, 43, L588. [CrossRef]

18. Parkin, S.S.P.; Kaiser, C.; Panchula, A.; Rice, P.M.; Hughes, B.; Samant, M.; Yang, S.-H. Giant tunnelling magnetoresistance at room temperature with $\mathrm{MgO}$ (100) tunnel barriers. Nat. Mater. 2004, 3, 862. [CrossRef]

19. Hong, J.; Wu, R.Q.; Lindner, J.; Kosubek, E.; Baberschke, K. Manipulation of Spin Reorientation Transition by Oxygen Surfactant Growth: A Combined Theoretical and Experimental Approach. Phys. Rev. Lett. 2004, 92, 147202. [CrossRef] 
20. Hillebrecht, F.U.; Fuggle, J.C.; Bennett, P.A.; ZoInierek, Z.; Freiburg, C. Electronic structure of Ni and Pd alloys. II. X-ray photoelectron core-level spectra. Phys. Rev. B 1983, 27, 2179. [CrossRef]

21. Dzyaloshinskii, L.E. Thermodynamic Theory of "Weak" Ferromagnetism in Antiferromagnetic Substances. Sov. Phys. JIETP 1957, $5,1259$.

22. Moriya, T. Anisotropic Superexchange Interaction and Weak Ferromagnetism. Phy. Rev. 1960, 120, 91. [CrossRef]

23. Bychkov, Y.A.; Mel'nikov, V.I.; Rashba, É. Effect of spin-orbit coupling on the energy spectrum of a 2D electron systems in a tilted magnetic field. Zh. Eksp. Theor. Fiz. 1990, 98, 717.

24. Chikazumi, S. Physics of Ferromagnetism; Clarendon: Oxford, UK, 1997.

25. Pateras, A.; Harder, R.; Manna, S.; Kiefer, B.; Sandberg, R.L.; Trugman, S.; Kim, J.W.; de la Venta, J.; Fullerton, E.E.; Shpyrko, O.G.; et al. Room temperature giant magnetostriction in single-crystal nickel nanowires. NPG Asia Mater. 2019, 11, 59. [CrossRef]

26. Hillebrands, B.; Ounadiela, K.E. Spin Dynamics in Confined Magnetic Structures; Springer: Berlin/Heidelberg, Germany, 2002; Volume I-III.

27. Schrefl, T.; Fidler, J.; Kirk, K.J.; Chapman, J.N. Domain structures and switching mechanisms in patterned magnetic elements. J. Magn. Magn. Mater. 1997, 175, 193. [CrossRef]

28. Shigeto, K.; Shinjo, T.; Ono, T. Injection of a magnetic domain wall into a submicron magnetic wire. Appl. Phys. Lett. 1999, 75, 2815. [CrossRef]

29. Cowburn, R.P.; Gray, S.J.; Ferré, J.; Bland, J.A.C.; Miltat, J. Magnetic switching and in-plane uniaxial anisotropy in ultrathin Ag/Fe/Ag(100) epitaxial films. J. Appl. Phys. 1995, 78, 7210. [CrossRef]

30. Yamaguchi, A.; Ono, T.; Nasu, S.; Miyake, K.; Mibu, K.; Shinjo, T. Real-Space Observation of Current-Driven Domain Wall Motion in Submicron Magnetic Wires. Phys. Rev. Lett. 2004, 92, 077205. [CrossRef]

31. Dean, J.; Bryan, M.T.; Cooper, J.D.; Virbule, A.; Cunningham, J.E.; Hayward, T.J. A sound idea: Manipulating domain walls in magnetic nanowires using surface acoustic waves. Appl. Phys. Lett. 2015, 107, 142405. [CrossRef]

32. Ostler, T.A.; Cuadrado, R.; Chantrell, R.W.; Rushforth, A.W.; Cavill, S.A. Strain Induced Vortex Core Switching in Planar Magnetostrictive Nanostructures. Phys. Rev. Lett. 2015, 115, 067202. [CrossRef]

33. Yamaguchi, A.; Ohkouchi, T.; Yasui, A.; Kinoshita, T.; Yamada, K. Control of Domain Structure in Artificial Ni Wires Fabricated on an $\mathrm{LiNbO}_{3}$ Substrate. IEEE Trans. Magn. 2017, 53, 8108504. [CrossRef]

34. Yamaguchi, A.; Ohkouchi, T.; Yasui, A.; Kinoshita, T.; Yamada, K. Heterojunction-induced magnetic anisotropy and magnetization reversal of $\mathrm{Ni}$ wires on $\mathrm{LiNbO}_{3}$ substrate. J. Magn. Magn. Mater. 2018, 453, 107. [CrossRef]

35. Yamaguchi, A.; Yamada, K.; Nakao, A.; Saiki, T.; Utsumi, Y.; Ogasawara, T. The Study on Magnetization Reversal of Stripe-Domain Structure in Ni Wires Fabricated on a $\mathrm{LiNbO}_{3}$ Substrate. IEEE Trans. Magn. 2019, 55, 2500404. [CrossRef]

36. Nakamura, R.; Saegusa, S.; Suzuki, S.; Nakao, A.; Utsumi, Y.; Ohkochi, T.; Oura, M.; Takizawa, Y.; Saiki, T.; Lee, T.; et al. Magnetic Scattering in Ni Wires Fabricated on Ferroelectric $\mathrm{LiNbO}_{3}$ Substrate for Magnetic Sensor Application. Sens. Mater. 2019, $31,3007$. [CrossRef]

37. Foerster, M.; Aballe, L.; Hernàndez, J.M.; Macià, F. Subnanosecond magnetization dynamics driven by strain waves. MRS Bull. 2019, 43, 854. [CrossRef]

38. Foerster, M.; Macià, F.; Statuto, N.; Finizio, S.; Hernàndez-Mínguez, A.; Lendínez, S.; Santos, P.V.; Fontcuberta, J.; Hernàndez, J.M.; Kläi, M.; et al. Direct imaging of delayed magneto-dynamic modes induced by surface acoustic waves. Nat. Commun. $2019,8,407$. [CrossRef]

39. Guo, F.Z.; Muro, T.; Matsushita, T.; Wakita, T.; Ohashi, H.; Senba, Y.; Kinoshita, T.; Kobayashi, K.; Saitoh, Y.; Koshikawa, T.; et al. Characterization of spectroscopic photoemission and low energy electron microscope using multipolarized soft $\mathrm{x}$ rays at BL17SU/SPring-8. Rev. Sci. Instrum. 2007, 78, 066107. [CrossRef]

40. Tanuma, S.; Powell, C.J.; Penn, D.R. Calculation of electron inelastic mean free paths (IMFPs) VII. Reliability of the TPP-2M IMFP predictive equation. Surf. Interf. Anal. 1993, 21, 165. [CrossRef]

41. NIST X-ray Photoelectron Spectroscopy Database. Available online: https://srdata.nist.gov/xps/ (accessed on 14 April 2021).

42. Vakulov, Z.; Zamburg, E.; Khajhulin, D.; Geldash, A.; Golosov, D.A.; Zavadski, S.M.; Miakonkikh, A.V.; Rudenko, K.V.; Dostanko, A.P.; He, Z.; et al. Oxygen Pressure Influence on Properties of Nanocrystalline $\mathrm{LiNbO}_{3}$ Films Grown by Laser Ablation. Nanomaterials 2020, 10, 1371. [CrossRef] 